\title{
A PROCEDURE FOR DETERMINATION OF THE EXPONENTIAL STABILITY OF CERTAIN DIFFERENTIAL-DIFFERENCE EQUATIONS*
}

$\mathrm{BY}$

R. DATKO

Georgetown University

\begin{abstract}
Certain continuity properties of the spectra of linear autonomous differential-difference equations which depend on a parameter are developed. These results are used to obtain a practical criterion for determination of the exponential stability of these systems.
\end{abstract}

Introduction. In this paper a problem arising from the study of linear differential difference equations is considered. Suppose we are given an $n$-dimensional family of differential difference equations of the type

$$
\frac{d}{d t}\left[x(t)-\sum_{j=1}^{m} B_{j} x\left(t-\alpha h_{j}\right)\right]=\sum_{j=0}^{m} A_{j} x\left(t-\alpha h_{j}\right), \quad \alpha \geq 0,
$$

where $0=h_{0}<h_{1}<h_{2}<\cdots<h_{m}$ are fixed constants. We ask the questions: "In what manner do the stability properties of $(0.1)$ depend on a change in $\alpha$ ? In particular, if $(0.1)$ is asymptotically stable for $\alpha=0$, how large can we take $\alpha$ and still preserve this property?"

In the case where $B_{j}=0$ for all $j$, i.e. the retarded case, the answer to this last question is quite easily given. In this case the roots of the characteristic equation (see e.g. [5]), although infinite in number, possess a continuity property which allows one to determine or at least approximate the size of the largest $q$ for which $\alpha \varepsilon[0, q)$ guarantees that the corresponding system $(0.1)$ is asymptotically stable. If $B_{j} \neq 0$ for some $j$, the above questions become more difficult. The procedure used in this paper to study these questions may be outlined as follows. For each $\alpha$ in $[0, \infty)$ we define $\sigma(\alpha)=$ sup $\operatorname{Re}\{\mathrm{s}: s$ is in the point spectrum of $(0.1)\}$. Then for $\alpha>0$ we can prove that $\sigma(\alpha)$ is continuous. In the retarded case $\sigma(\alpha)$ is also continuous at $\alpha=0$. In the general case $\sigma(\alpha)$ is continuous at $\alpha=0$ for a particular class of systems which contains the uniformly exponentially stable systems. Thus classes of systems $(0.1)$ for which $\sigma(\alpha)$ is continuous on $[0, \infty)$ provide us with a method of determining whether for a given $\alpha_{0}$ the system is uniformly asymptotically stable. For if $\sigma(0)<0$ and $\alpha_{0} \in[0, q)$, where $q$ is the smallest number which satisfies $\sigma(\alpha)$ $=0$, then $\sigma\left(\alpha_{0}\right)<0$. But from the general theory of retarded and neutral functional equations (see e.g. [6] and [7] we know that $\sigma\left(\alpha_{0}\right)<$ guarantees that for the value $\alpha_{0}$ $(0.1)$ is uniformly exponentially stable.

The idea of studying stability though examination of the manner in which the point spectrum of $(0.1)$ varies as $\alpha$ varies is similar to the method of $D$-partitions used to study the stability of retarded linear systems. A good outline of this method can be found in $[5$,

\footnotetext{
* Received September 8. 1977.
} 
p. 132]. The results in this paper are an outgrowth of a report [4] on the stabilization of linear functional differential equations. In particular, Theorems 2.2 and 2.3 represent a considerable extension of the work reported there.

\section{Preliminaries.}

Notation. 1. The symbols $B_{j}, j=1, \cdots m$ and $A_{j}, j=0,1, \cdots m$ will denote specific complex $n \times n$ matrices, $I$ will denote the $n \times n$ identity matrix. The norm, $|B|$, of any $n \times$ $n$ matrix $B=\left\{b_{i j}\right\}$ will be defined to be $\sup _{i, j}\left\{\left|b_{i j}\right|\right\}$. An $n \times n$ real or complex matrix $B$ will be said to be Hurwitzian if the real parts of its eigenvalues all lie in a half plane $\operatorname{Re} z<$ $-\beta, \beta>0$. The Euclidean norm of any complex $n$-vector $x$ will be denoted by $|x|$.

2. $X$ will stand for the Banach space of all continuous mappings from an interval $[-h$, $0]$ into $R^{n}$, the Euclidean $n$-space. The norm $X$ is given by $|\phi|=\{\sup |\phi(t)|: t \in[-h, 0]\}$.

3. $R^{+}$will denote the nonnegative half line $[0, \infty)$. The complex plane will be denoted by $C$. An infinite vertical strip in the complex plane of the form $\{\mathrm{s}: a<\operatorname{Re} s<b\}$ will be denoted by $(a, b)$. If the strip is closed, i.e. we consider $\{\mathrm{s}: a \leq \operatorname{Re} s \leq b\}$, we write $[a, b]$. If $b=+\infty$, the strip is called a right half plane (open or closed as the case may be), if $a=-$ $\infty$, a left half plane.

Although we shall also use $(a, b)$ and $[a, b]$ for open and closed intervals of $R^{1}$, we believe no confusion will arise since the context will indicate whether we are considering a vertical strip or an interval. The reason for the above notation for vertical strips in the complex plane is that it is common usage in much of the work involving almost periodic functions (see e.g. [1]).

4. Let $Q(s)$ be an $n \times n$ complex matrix dependent on the complex variable $s$. We define $\sigma(Q)=\sup \{\operatorname{Re} s: \operatorname{det}(Q(s))=0\}$, if $\operatorname{det}(Q(s)) \neq 0$ for at least one finite value of $s$. If $\operatorname{det}(Q(s)) \neq 0$ for all finite $s$ we define $\sigma(Q)=-\infty$. In either case we shall, from now on, refer to $\sigma(Q)$ as the spectral limit of the matrix function $Q$.

2. The parametric dependence of the spectral limit of certain matrix functions. Let $\alpha$ be in $R^{+}$and

$$
0=h_{0}<h_{1}<\cdots<h_{m}=h .
$$

Consider the matrix function

$$
F_{\alpha}(s)=\left[s I-\sum_{j=0}^{m} A_{j} \exp \left(-\alpha s h_{j}\right)\right] .
$$

THEOREM 2.1. For each $\alpha$ in $R^{+} \sigma\left(F_{\alpha}\right)$ is finite and defines a continuous function as $\alpha$ varies.

Proof. (i) It can be shown (see e.g. [6]) from the general theory of autonomous functional differential equations that for each $\alpha$ in $R^{+} \operatorname{det}\left(F_{\alpha}(s)\right)=0$ has a solution in $C$ and that $\sigma\left(F_{\alpha}\right)<\infty$ for each $\alpha$. (ii) Proof of continuity. Again from the general theory of functional differential equations, it is known that given $\alpha_{0}$ in $R^{+}$there exists $s_{0}$ in $C$ such that $\operatorname{det}\left(F_{\alpha_{0}}(s)\right)=0$ and $\sigma\left(F_{\alpha_{0}}\right)=\operatorname{Re} s_{0}$. Suppose $\left\{\alpha_{n}\right\} \subset R^{+}$tends to $\alpha_{0}$. By Hurwitz' theorem [8] there exists $\left\{s_{n}\right\} \subset C$ such that $\left\{s_{n}\right\}$ tends to $s_{0}$ and for each $n \operatorname{det} F_{\alpha_{n}}\left(s_{n}\right)=0$. Since, by definition, $\operatorname{Re} s_{n} \leq \sigma\left(F_{\alpha_{n}}\right)$ it follows that $\varliminf^{\lim } \sigma\left(F_{\alpha_{n}}\right) \geq \operatorname{Re} s_{0}=\sigma\left(F_{\alpha_{0}}\right)$.

We shall now demonstrate that $\overline{\lim } \sigma\left(F_{\alpha_{n}}\right) \leq \sigma\left(F_{\alpha_{0}}\right)$ and thus prove continuity at $\alpha_{0}$ and hence, since $\alpha_{0}$ is arbitrary, continuity on $R^{+}$. The demonstration is by contradiction.

Suppose there exists $\epsilon_{0}>0$ and a subsequence $\left\{\alpha_{q}\right\} \not \subset\left\{\alpha_{n}\right\}$ such that for all $\alpha_{q} \sigma\left(F_{\alpha_{q}}\right) \geq$ 
$\sigma\left(F_{\alpha_{0}}\right)+\epsilon_{0}$. We may also assume without loss that all $\alpha_{q}$ satisfy

$$
\alpha_{0}-\left(\epsilon_{0} / 2\right) \leq \alpha_{q} \leq \alpha_{0}+\left(\epsilon_{0} / 2\right) \text {. }
$$

Let

$$
L_{1}=\left\{s \in C: 2 \sum_{j=0}^{m}\left|A_{j}\right| \exp \left[-\left(\alpha_{0}-\frac{\epsilon_{0}}{2}\right) \sigma\left(\alpha_{0}\right) h_{j}\right] \geq|s| \text { and } \operatorname{Re} s \geq s_{0}+\frac{\epsilon_{0}}{2}\right\} .
$$

Let $s_{1}>\sigma\left(\alpha_{0}\right)+2 \epsilon_{0}$ be such that

$$
s_{1}>\sum_{j=0}^{m}\left|A_{j}\right| \exp \left[-s_{1}\left(\alpha_{0}-\left(\epsilon_{0} / 2\right)\right) h_{j}\right]
$$

and define

$$
L_{2}=\left\{s \in C: \operatorname{Re} s \leq s_{1}\right\} .
$$

If $K=L_{1} \cap \mathrm{L}_{2}$, then $K$ is compact and by our construction all the zeros of $\operatorname{det} F_{\alpha_{q}}(s)=0$ with $\operatorname{Re} s \geq s_{0}+\epsilon_{0} / 2$ must lie in $K$. Thus to each index $\alpha_{q}$ there corresponds at least one point $s_{q}$ in $K$ such that $\operatorname{det} F_{\alpha_{q}}\left(s_{q}\right)=0$. Moreover, on $K$ it is trivial to prove, since $\left\{\alpha_{q}\right\}$ tends to $\alpha_{0}$, that $\left\{\right.$ det $\left.F_{\alpha_{q}}(s)\right\}$ converges uniformly to $\operatorname{det} F_{\alpha_{0}}(s)$. Thus let $\left\{s_{r}\right\} \subset\left\{s_{q}\right\}$ be a convergent subsequence with limit $s_{2}$. Then it follows that $\operatorname{Re} s_{2} \geq \sigma\left(F_{\alpha}\right)+\epsilon_{0} / 2$ and

$$
\lim _{r \rightarrow \infty} \operatorname{det} F_{\alpha_{r}}\left(s_{r}\right)=0=\operatorname{det} F_{\alpha_{0}}\left(s_{2}\right) .
$$

But then $\sigma\left(F_{\alpha_{0}}\right) \geq \operatorname{Re} s_{2} \geq \sigma F\left(\alpha_{0}\right)+\epsilon_{0} / 2$, which is impossible. This contradiction proves that $\lim \sigma F\left(\alpha_{n}\right) \leq \sigma\left(F_{\alpha_{0}}\right) \leq \lim F\left(\alpha_{0}\right)$ and hence that $\sigma\left(F_{\alpha}\right)$ is continuous at $\alpha_{0}$ and consequently on $R^{+}$.

We next wish to consider matrix functions of the type

$$
G_{\alpha}(s)=s\left(I-\sum_{j=1}^{m} B_{j} \exp \left(-\alpha s h_{j}\right)\right)-\sum_{j=0}^{m} A_{j} \exp \left(-\alpha s h_{j}\right), \alpha \in R^{+} .
$$

The continuity of $\sigma\left(G_{\alpha}\right)$ is a more difficult problem for these functions and will require some preliminary lemmas. First let us define the sequence of points $\left\{\omega_{n}\right\} \subset R^{+}$as follows. Let

$$
h_{1}=\omega_{1}<\omega_{2}<\cdots \omega_{n}<\cdots
$$

where, for each $n, \omega_{n}$ is a linear combination of the form

$$
\omega_{n}=n_{1} h_{1}+n_{2} h_{2}+\cdots+n_{m} h_{m}
$$

with $n_{j}, 1 \leq j \leq m$, either zero or a positive integer.

Lemma 2.1. Assume $\left\{H_{j}\right\}$ is a sequence of real $n \times n$ matrices such that for some $\delta, \sum_{j=1}^{\infty}$ $\left|H_{j}\right| \exp \left(-\delta \omega_{j}\right)<\infty$. Let $\alpha_{0}>0$ and $\eta>0$. Define

$$
A_{\alpha}(s)=s I-\sum_{j=1}^{\infty} H_{j} \exp \left(-\alpha s \omega_{j}\right)
$$

and assume that

$$
\sigma\left(s I-\sum_{j=1}^{\infty} H_{j} \exp \left(-\alpha_{0} s \omega_{j}\right)\right)=\frac{\delta}{\alpha_{0}}+\eta .
$$

Then $\sigma\left(A_{\alpha}\right)$ is continuous at $\alpha_{0}$. 
Proof. We shall restrict ourselves to neighborhoods of $\alpha_{0}$ depending upon whether $\delta<$ $0, \delta=0$ or $\delta>0$.

(i) If $\delta<0$ we consider only those $\alpha$ for which $\left|\alpha_{0}-\alpha\right|<\alpha_{0}^{2} \eta / 2|\delta|$.

(ii) If $\delta=0$ all $\alpha>0$ will suffice.

(iii) If $\delta>0$ we consider only $\alpha$ such that $\alpha>\alpha_{0} / 2$ and $\left|\alpha_{0}-\alpha\right|<\alpha_{0}^{2} \eta / 4 \delta$.

We do this to ensure that for this range of $\alpha \sum_{j=1}^{\infty}\left|H_{j}\right| \exp \left(-\alpha \omega_{j} \operatorname{Re} s\right)<\infty$ if $\operatorname{Re} s \geq$ $\left(\delta / \alpha_{0}\right)+(\eta / 2)$.

Proceeding as in the proof of Theorem 2.1, we can show using Hurwitz' theorem [8] that if $\alpha_{0}=\lim _{n \rightarrow \infty} \alpha_{n}$ then $\lim \sigma\left(A_{\alpha_{n}}\right) \geq \sigma\left(A_{\alpha_{0}}\right)$. We then show that $\overline{\lim } \sigma\left(A_{\alpha_{n}}\right) \leq \sigma\left(A_{\alpha_{0}}\right)$ by assuming the contrary. By our restrictions on $\alpha$ we know that $\operatorname{det}\left[s I-\sum_{j=1}^{\infty} H_{j} \exp \right.$ $\left.\left(-\alpha \omega_{j} s\right)\right]$ converges absolutely if $\operatorname{Re} s \geq\left(\delta / \alpha_{0}\right)+(\eta / 2)$. Thus if $\overline{\lim } \sigma\left(A_{\alpha_{n}}\right)>\sigma\left(A_{\alpha_{0}}\right)$ we can argue, as was done in proving Theorem 2.1, that there exists $\epsilon_{0}>0$ and a compact set $K \subset$ $C$ such that $K \subset\left\{s: \operatorname{Re} s \geq\left(\delta / \alpha_{0}\right)+\eta+\left(\epsilon_{0} / 2\right)\right\}$ and $\operatorname{det}\left[s_{2} I-\sum_{j=1}{ }^{\infty} H_{j} \exp \left(-\alpha_{0} \omega_{j} s_{2}\right)\right]=0$ for some $s_{2}$ in $K$. This is a contradiction. Hence $\lim \sigma\left(A_{\alpha_{n}}\right) \leq \sigma\left(A_{\alpha_{0}}\right) \leq \underline{\lim } \sigma\left(A_{\alpha_{n}}\right)$, which proves the continuity of $\sigma\left(A_{\alpha}\right)$ at $\alpha_{0}$.

The next lemma is a modification of Rouché's theorem [8]. It is stated without proof, since its proof is basically the same as that of the original (see e.g. [8]).

Lemma 2.2. For each $\lambda \in[0,1]$ let $f(s, \lambda)$ and $(\partial f / \partial s)(s, \lambda)$ be analytic is $s$ in some region $G$ of the complex plane and uniformly continuous on $G \times[0,1]$. Let $J$ be a closed Jordan curve contained in $G$ such that $|f(s, \lambda)| \geq \epsilon>0$ for $(s, \lambda) \in J \times[0,1]$. Then, inside of $J, f(s, 0)$ and $f(s, 1)$ have the same number of zeroes counting their multiplicities.

Let

$$
B(s)=I-\sum_{j=1}^{m} B_{j} \exp \left(-s h_{j}\right) .
$$

Observe that $B(s)$ is an almost-periodic analytic matrix function. One property of such functions is that if $|B(s)|$ is uniformly bounded on an open vertical strip $\left(s_{0}-\delta, s_{0}+\delta\right), \delta$ $>0$, and if $\epsilon>0$ is given there exists $l(\epsilon)$ such that every open interval of length $l(\epsilon)$ contains a point $\tau$ such that $|B(s)-B(s+i \tau)| \leq \epsilon$ for all $s \in\left(s_{0}-\delta, s_{0}+\delta\right)$ (see e.g. [1, pp. 141-143]). This in turn implies the existence of a sequence $\left\{\tau_{n}\right\}$ such that $1+\tau_{n} \leq \tau_{n+1}$ for all $n$ and such that

$$
\left|B(s)-B\left(s+i \tau_{n}\right)\right| \leq \epsilon
$$

for all $n$ and all $s \in\left(s_{0}-\delta, s_{0}+\delta\right)$.

Let

$$
A(s)=\sum_{j=0}^{m} A_{j} \exp \left(-s h_{j}\right)
$$

and

$$
G(s)=B(s)-\frac{1}{s} A(s) .
$$

Lemma 2.3. If $s_{0}+i \tau_{0}$ is a zero of $\operatorname{det} B(s)$, then for any $\delta>0$ the open vertical strip $\left(s_{0}-\delta\right.$, $\left.s_{0}+\delta\right)$ contains an infinite number of zeros of both det $B(s)$ and det $G(s)$.

Proof. We shall first show that $\left(s_{0}-\delta, s_{0}+\delta\right)$ contains an infinite number of zeros of $\operatorname{det} B(s)$. Since det $B(s)$ is a nonzero entire function in the complex plane we can find $\eta$ such that $0<\eta<\delta / 2$ and $\eta<1$, and an $\epsilon>0$ such that $|\operatorname{det} B(s)| \geq \epsilon$ on the circle $\mid s_{0}+i \tau_{0}-$ $z \mid=\eta$. Also, because $\operatorname{det} B(s)$ is a uniformly bounded almost periodic function on $\left(s_{0}-\delta\right.$, 
$\left.s_{0}+\delta\right)$, there exists a sequence of real numbers $\left\{\tau_{n}\right\}$ such that $1 \leq \tau_{1}, \tau_{n}+1 \leq \tau_{n+1}$ for all $n$ and for all $n$ and $s \in\left(s_{0}-\delta, s_{0}+\delta\right)$

$$
\left|\operatorname{det} B\left(s+i \tau_{n}\right)-\operatorname{det} B(s)\right| \leq \epsilon / 4 \text {. }
$$

We claim that in each disc of the form

$$
K\left(s_{0}+i\left(\tau_{0}+\tau_{n}\right), \eta\right)=\left\{s:\left|s_{0}+i\left(\tau_{0}+\tau_{n}\right)-s\right| \leq \eta\right\}
$$

there is a zero of $\operatorname{det} B(s)$.

Suppose the contrary. Then for some $n$ and all $\left.s \in K\left(s_{0}+i \tau_{0}+\tau_{n}\right), \eta\right)$ g.l.b. $|\operatorname{det} B(s)|$ $=m>0$ where, $m \leq \epsilon / 4$. From the theory of complex variables it follows that $|\operatorname{det} B(s)|$ attains its minimum value at some point $s_{1}$ on the boundary of $K\left(s_{0}+i\left(\tau_{0}+\tau_{n}\right), \eta\right)$. But by our construction $s_{1}$ must be of the form $s_{1}=\hat{s}+i \tau_{n}$ where $\left|\hat{s}-\left(s_{0}+i \tau_{0}\right)\right|=\eta$. We then obtain the inequality

$$
\begin{aligned}
\epsilon & \leq|\operatorname{det} B(\hat{s})| \leq\left|\operatorname{det} B\left(\hat{s}+i \tau_{n}\right)-\operatorname{det} B(\hat{s})\right|+\left|\operatorname{det} B\left(\hat{s}+i \tau_{n}\right)\right| \\
& \leq \frac{\epsilon}{4}+m \leq \frac{\epsilon}{2}
\end{aligned}
$$

which is a contradiction. Hence det $B(s)$ has at least one zero in $K\left(s_{0}+i\left(\tau_{0}+\tau_{n}\right), \eta\right)$. This proves the first conclusion of the lemma.

To prove that det $G(s)$ has an infinite number of zeros in $\left(s_{0}-\delta, s_{0}+\delta\right)$, observe that for $n$ sufficiently large $|\operatorname{det}(B(s)-(\lambda / \mathrm{s}) A(s))| \geq \in / 4$ uniformly for $\in \epsilon[0,1]$ if $s \in\{s$ : $\left.\left|s_{0}+i\left(\tau_{0}+\tau_{n}\right)-s\right|=\eta\right\}$. Thus by Lemma $2.2 \operatorname{det} B(s)$ and $G(s)$ have the same number of zeros in the disc $K\left(s_{0}+i\left(\tau_{0}+\tau_{n}\right), \eta\right)$ if $n$ is sufficiently large. This completes the proof of the lemma.

The formal Dirichlet series obtained by inversion of $B(s)$ in $(2.7)$ is of the form

$$
B^{-1}(s) \sim I+\sum_{j=1}^{\infty} \hat{H}_{j} \exp \left(-\omega_{j} s\right)
$$

where $\left\{\omega_{j}\right\}$ are defined by (2.4). The wiggle in (2.12) denotes the Fourier representation of $B^{-1}(s)$ (see e.g. [1]). However, in any closed right half plane of the complex plane in which $\operatorname{det}(B(s))$ has no zeros this convergence is absolute in the sense that

$$
\left|B^{-1}(s)\right| \leq 1+\sum_{j=1}^{\infty}\left|\hat{H}_{j}\right| \exp \left(-\omega_{j} \operatorname{Re} s\right)>\infty .
$$

This statement is the content of the following lemma.

LEMma 2.4. The Dirichlet series of $B^{-1}(s)$ given by (2.12) converges absolutely in any closed right half plane in which $B^{-1}(s)$ is uniformly bounded. Hence if $\left(s_{0}, \infty\right)$ is free of zeros of det $B(s)$, then given any $\epsilon>0, B^{-1}(s)$ converges absolutely in $\left[s_{0}+\epsilon, \infty\right)$.

Proof. It is shown in [3] that the sequence $\left\{\omega_{j}\right\}$ defined by (2.4) has the property that $\lim _{n \rightarrow \infty}\left(\ln n / \omega_{n}\right)=0$. Since $\omega_{n}>0$ for all $n$, a result due to Bohr [2] states that under these conditions the Dirichlet series of $B^{-1}(s)$ converges absolutely in any closed right half plane in which it is uniformly bounded. This proves the lemma.

LEMma 2.5. Let $\alpha>0$. Then the set of zeros of

$$
\operatorname{det} B_{\alpha}(s)=\operatorname{det}\left[I-\sum_{j=1}^{m} B_{j} \exp \left(-\alpha s h_{j}\right)\right],
$$

if they exist, is the set of zeros of $\left\{s_{0}{ }^{n} / \alpha\right\}$ where $\left\{s_{0}{ }^{n}\right\}$ is the set of zeros of det $B(s)$. 
Conversely, if $\left\{s_{\alpha}{ }^{n}\right\}$ denotes the set of zeros of (2.13) then the set of zeros of det $B(s),\left\{s_{0}{ }^{n}\right\}$, satisfies for each $n$ the relation

$$
s_{0}^{n}=\alpha s_{\alpha}^{n} .
$$

Proof. The proof is obtained by direct substitution.

THEOREM 2.2. The matrix function

$$
G_{\alpha}(s)=\left[s\left(I-\sum_{j=1}^{m} B_{j} \exp \left(-\alpha s h_{j}\right)\right)-\sum_{j=0}^{m} A_{j} \exp \left(-\alpha s h_{j}\right)\right]
$$

has a spectral limit function $\sigma\left(G_{\alpha}\right)$ which is continuous on $(0, \infty)$.

Proof. To prove this theorem we shall show that $\sigma\left(G_{\alpha}\right)$ is continuous at an arbitrary point $\alpha_{0}$ in $(0, \infty)$. Thus let $\alpha_{0}>0$ be fixed. Notice that one consequence of Lemmas 2.3 and 2.5 is that $\sigma\left(G_{\alpha_{0}}\right) \geq \sigma(B) / \alpha_{0}$. We consider two cases: (i) $\sigma\left(G_{\alpha_{0}}\right)>\sigma(B) / \alpha_{0}$ or (ii) $\sigma\left(G_{\alpha}\right)$ $=\sigma(B) / \alpha_{0}$.

Case (i). There exist $\delta_{0}>0$ such that $\sigma\left(G_{\alpha}\right)=\left(\sigma(B) / \alpha_{0}\right)+\delta_{0}$. Hence by Lemma 2.4 the Dirichlet series

$$
\left[I-\sum_{j=1}^{m} B_{j} \exp \left(-\alpha_{0} s h_{j}\right)\right]^{-1}=I+\sum_{k=1}^{\infty} \hat{H}_{j} \exp \left(-\alpha_{0} \omega_{k} s\right)
$$

converges absolutely in the closed half plane $\left[\left(\sigma(B) / \alpha_{0}\right)+\left(\delta_{0} / 2\right), \infty\right)$. Furthermore, det $G_{\alpha_{0}}(s)=0$ has solutions in this closed half plane. Thus $\sigma\left(G_{\alpha_{0}}\right)$ is determined by the expression

$$
\begin{aligned}
G_{\alpha_{0}}(s)\left[I-\sum_{j=1}^{m}\right. & \left.B_{j} \exp \left(-\alpha_{0} h_{j} s\right)\right]^{-1} \\
= & s I-\left(\sum_{j=0}^{m} A_{j} \exp \left(-\alpha_{0} s h_{j}\right)\right)\left(I+\sum_{k=1}^{\infty} \hat{H}_{k} \exp \left(-\alpha_{0} \omega_{k} s\right)\right) \\
= & s I-A_{0}-\sum_{k=1}^{\infty} H_{k} \exp \left(-\alpha_{0} \omega_{k} s\right)=A_{\alpha_{0}}(s),
\end{aligned}
$$

where $A_{\alpha_{0}}(s)$ is absolutely convergent in the half plane $\left[\left(\sigma(B) / \alpha_{0}\right)+\left(\delta_{0} / 2\right), \infty\right)$. Thus if $\alpha>0$,

$$
s I-A_{0}-\sum_{k=1}^{\infty} H_{k} \exp \left(-\alpha \omega_{k} s\right)=A_{\alpha}(s)
$$

is absolutely convergent in the half plane

$$
\left[\frac{\alpha_{0}}{\alpha}\left(\frac{\sigma(B)}{\alpha_{0}}+\frac{\delta_{0}}{2}\right), \infty\right) .
$$

Also notice that det $A_{\alpha}(s), \alpha>0$, can have at most a finite number of zeros in the right half plane (2.19). This is because in the closed right half plane (2.19) $\mid$ det $A_{\alpha}(s) \mid$ tends to infinity as $|s|$ tends to infinity. We also know that by assumption det $A_{\alpha_{0}}(s)$ has at least one zero in the plane $\left[\left(\sigma(B) / \alpha_{0}\right)+\left(\delta_{0} / 2\right), \infty\right)$. Hence by Lemma $2.1 \sigma\left(A_{\alpha}\right)$ is continuous at $\alpha_{0}$. Let $B_{\alpha}$ be defined by (2.13) and notice that by (2.14) $\sigma\left(B_{\alpha}\right)=\sigma\left(B_{1}\right) / \alpha$ if $\alpha>0$. Hence $\sigma\left(B_{\alpha}\right)$ is continuous on $(0, \infty)$. Thus it follows that for $\alpha$ sufficiently close to $\alpha_{0} \sigma\left(G_{\alpha}\right)=$ $\sigma\left(A_{\alpha}\right)$. Hence

$$
\lim _{\alpha \rightarrow \alpha_{0}} \sigma\left(G_{\alpha}\right)=\lim _{\alpha \rightarrow \alpha_{0}} \sigma\left(A_{\alpha}\right)=\sigma\left(A_{\alpha_{0}}\right)
$$


which proves continuity if $\sigma\left(G_{\alpha_{0}}\right)>\sigma\left(B_{\alpha}\right)$.

Case (ii). Assume $\sigma\left(G_{\alpha_{0}}\right)=\sigma\left(B_{\alpha_{0}}\right)$. Again by (2.14) we know that $\sigma\left(B_{\alpha}\right)$ is continuous at $\alpha_{0}$. Thus if $\left\{\alpha_{n}\right\}$ tends to $\alpha_{0}, \lim \sigma\left(G_{\alpha}\right) \geq \sigma\left(B_{\alpha_{0}}\right)=\lim _{\alpha \rightarrow \alpha_{0}} \sigma\left(B_{\alpha}\right)$.

Assume $\lim \sigma\left(G_{\alpha}\right)>\sigma\left(G_{\alpha_{0}}\right)$. Then there exists $\epsilon_{0}>0$ such that

$$
\sigma\left(G_{\alpha_{0}}\right)+\epsilon_{0} \leq \varlimsup \lim \sigma\left(G_{\alpha_{n}}\right) .
$$

But then in the closed half plane $\left[\sigma\left(B_{\alpha_{0}}\right)+\epsilon_{0} / 2, \infty\right)$ Eqs. (2.16) and (2.18) converge absolutely, and using (2.19) we see that for $\alpha$ sufficiently close to $\alpha_{0}$ (2.18) converges absolutely in the half plane $\left[\sigma\left(B_{\alpha_{0}}\right)+\left(\epsilon_{0} / 2\right)\right.$, $\left.\infty\right)$. Since $\overline{\lim } \sigma\left(G_{\alpha}\right) \geq \sigma\left(B_{\alpha_{0}}\right)+\epsilon_{0}$, det $A_{\alpha_{n}}(s)$ $=0$ has a solution in the closed half space $\left[\sigma\left(B_{\alpha_{0}}\right)+\frac{3}{4} \epsilon_{0}, \infty\right)$ for an infinite number of $\alpha_{n}$.

Again applying the arguments used in proving Theorem 2.1, we can find a compact set $K \in\left[\sigma\left(B_{\alpha_{0}}\right)+\frac{3}{4} \epsilon_{0}, \infty\right)$ such that for some $s_{1} \in K \operatorname{det} A_{\alpha_{0}}\left(s_{1}\right)=0$. But this leads to the contradiction

$$
\sigma\left(B_{\alpha_{0}}\right)+\frac{3}{4} \epsilon_{0} \sigma\left(G_{\alpha_{0}}\right)+\frac{3}{4} \epsilon_{0} \leq \operatorname{Re} s_{1} \leq \sigma\left(G_{\alpha_{0}}\right) .
$$

Hence $\overline{\lim } \sigma\left(G_{\alpha_{n}}\right) \leq \sigma\left(G_{\alpha_{0}}\right) \leq \lim \sigma\left(G_{\alpha_{n}}\right)$, which proves continuity at $\alpha_{0}$.

Example. In general, matrix functions of the type considered in Theorem 2.2 do not possess the property that $\sigma\left(G_{\alpha}\right)$ is continuous at $\alpha=0$. The following example illustrates this. Let

$$
G_{\alpha}(s)=(s+1)\left(1+\frac{1}{2} \exp \left(-\alpha \frac{\pi}{2} s\right)-\frac{1}{2} \exp (-\alpha \pi s)\right) .
$$

For $\alpha=0 \sigma\left(G_{0}\right)=1$. For $\alpha \neq 0, G_{\alpha}(2 i / \alpha)=0$ and $G_{\alpha}(s) \neq 0$ for $\operatorname{Re} s>0$. Hence for $\alpha \neq 0$ $\sigma\left(G_{\alpha}\right)=0$.

The following theorem states a condition which guarantees $\sigma\left(G_{\alpha}\right)$ is continuous at $\alpha=0$.

THEOREM 2.3. If

$$
\operatorname{det}\left[I-\sum_{j=1}^{m} B_{j} \exp \left(-s h_{j}\right)\right]=0
$$

has all roots lying in some left half plane $\left(-\infty,-\beta_{0}\right], \beta_{0}>0$, then $\sigma\left(G_{\alpha}\right)$ is continuous at $\alpha=0$.

Proof. Observe that if $\alpha>0$ then by (2.14) and the hypothesis of the theorem $\sigma\left(B_{\alpha}\right) \leq$ $-\beta_{0} / \alpha$. Consequently

$$
\lim _{\alpha \rightarrow 0^{+}} \sigma\left(B_{\alpha}\right)=-\infty .
$$

If $\operatorname{Re} s \geq-\beta_{0} / 2 \alpha$ we use (2.17) to obtain

$$
\begin{aligned}
s\left(I-\sum_{j=1}^{m} B_{j} \exp \left(-\alpha s h_{j}\right)\right) & -\sum_{j=0}^{m} A_{j} \exp \left(-\alpha s h_{j}\right) \\
= & {\left[s I-A_{0}-\sum_{j=1}^{\infty} H_{j} \exp \left(-\alpha s \omega_{j}\right)\right]\left[I-\sum_{j=1}^{m} B_{j} \exp \left(-\alpha s h_{j}\right)\right] } \\
= & A_{\alpha}(s) B_{\alpha}(s)=G_{\alpha}(s) .
\end{aligned}
$$


Because of (2.21) and (2.22) we see that on compact sets, $K$, in the complex plane

$$
\begin{array}{r}
\lim _{\alpha \rightarrow 0} A_{\alpha}(s) B_{\alpha}(s)=\left(s I-A_{0}-\sum_{j=1}^{\infty} H_{j}\right)\left(I-\sum_{j=1}^{m} B_{j}\right) \\
=s\left(I-\sum_{j=1}^{m} B_{j}\right)-\sum_{j=0}^{m} A_{j}
\end{array}
$$

and that this limit holds uniformly on $K$.

Using our version of Rouché's theorem (Lemma 2.2), (2.22) and (2.23), we conclude that if $\left\{\alpha_{n}\right\}, \alpha_{n}>0$ for all $n$, tends to zero there exists, for $n$ sufficiently large, $\left\{s_{n}\right\}$ in $C$ such that $\operatorname{det} A_{\alpha_{n}}\left(s_{n}\right)=0, \operatorname{Re} s_{n} / \alpha_{n} \geq-\beta_{0} / 2$ and $\lim _{n \rightarrow \infty} \operatorname{Re} s_{n}=\sigma\left(G_{0}\right)$. Hence for $n$ sufficiently large $\sigma\left(G_{\alpha_{n}}\right)=\sigma\left(A_{\alpha_{n}}\right)$ and $\varliminf \sigma\left(G_{\alpha_{n}}\right)=\varliminf \lim \sigma\left(A_{\alpha_{n}}\right) \geq \sigma\left(G_{0}\right)$.

If we assume that $\overline{\lim } \sigma\left(A_{\alpha_{n}}\right)>\sigma\left(G_{0}\right)$ we can repeat the type of argument used in the proofs of Theorem 2.1 and Lemma 2.1 to construct a compact set $K \subset C$ such that inf $\{\mathrm{Re}$ $s: s \in K\}>\sigma\left(G_{0}\right)$ and such that for some $s_{1} \in K \operatorname{det}\left[s_{1}\left(I-\sum_{j=1}^{m} B_{j}\right)-\sum_{j=0}{ }^{m} A_{j}\right]=0$. This contradiction proves that

$$
\overline{\lim } \sigma\left(A_{\alpha_{n}}\right) \leq \sigma\left(G_{0}\right) \leq \underline{\lim } \sigma\left(A_{\alpha_{n}}\right)
$$

and hence, since $\sigma\left(G_{\alpha_{n}}\right)=\sigma\left(A_{\alpha_{n}}\right)$ for $\alpha_{n}$ sufficiently small, that $\sigma\left(G_{\alpha}\right)$ is continuous at $\alpha=0$.

Remark 2.1. If $\sigma\left(G_{\alpha}\right)$ is determined by $\sigma\left(A_{\alpha}\right)$, there exists $s_{0}$ such that $\operatorname{Re} s_{0}=\sigma\left(G_{\alpha}\right)$. This is a consequence of the fact that in any right half plane in which $A_{\alpha}(s)$ is analytic, det $A_{\alpha}(s)=0$ can have at most a finite number of roots.

COROLlary to Theorem 2.3. The family

$$
\frac{d}{d t}\left[x(t)-B x\left(t-\alpha h_{m}\right)\right]=\sum_{j=0}^{m} A_{j} x\left(t-\alpha h_{j}\right), \quad \alpha \geq 0
$$

of differential-difference equations gives rise to a family of matrix functions $\left\{G_{\alpha}(s)\right\}$ such that $\sigma\left(G_{\alpha}\right)$ is continuous for all $\alpha \in[0, \infty)$ if $\operatorname{det}(\lambda I-B)=0$ has all roots in the disc $|\lambda|$ $<1$, i.e. $|\sigma(B)|<1$.

Proof. The point spectrum of (2.24) (see e.g. [6]) is obtained from the solutions of

$$
\operatorname{det}\left[s\left(I-B \exp \left(-\alpha s h_{m}\right)\right)-\sum_{j=0}^{m} A_{j} \exp \left(-\alpha s h_{m}\right)\right]=0,
$$

i.e. $\operatorname{det}_{\alpha}\left(G_{\alpha}(s)\right)=0$. By Theorem $2.2 \sigma\left(G_{\alpha}\right)$ is continuous for $\alpha>0$. The hypothesis of the corollary states that $\sigma\left(B_{1}\right)=\ln |\sigma(B)|<0$ if $\sigma(B) \neq 0$ and $\sigma\left(B_{1}\right)=-\infty$ if $\sigma(B)=0$.

Definition 2.1. An interval $[a, b)$ in $R^{+}$is an interval of exponential stability for the family of retarded differential-difference equations defined in $R^{n}$ by

$$
\frac{d}{d t}(x(t))=\sum_{j=0}^{m} A_{j} x\left(t-\alpha h_{j}\right),
$$

if (2.25) is exponentially stable for all $\alpha$ in $[a, b)$.

Similarly an interval $[a, b)$ in $R^{+}$is an interval of uniform exponential stability for the family of neutral differential-difference equations defined in $R^{n}$ by

$$
\frac{d}{d t}\left[x(t)-\sum_{j=1}^{m} B_{j} x\left(t-\alpha h_{j}\right)\right]=\sum_{j=0}^{m} A_{j} x\left(t-\alpha h_{j}\right),
$$


if (2.26) is uniformly exponentially stable for all $\alpha$ in $[a, b)$.

THEOREM 2.4. If on $[a, b) \sigma\left(F_{\alpha}\right)<0$ for every member of the family $(2.25)$, then $[a, b)$ is an interval of exponential stability for the family $(2.25)$.

Proof. The proof is a consequence of the general theory of functional differential equations [6], since $\sigma\left(F_{\alpha}\right)<0$ is a necessary and sufficient condition for a linear autonomous retarded functional differential equation to be exponentially stable.

Definition 2.2. A neutral differential-difference equation of the type (2.26) is said to be uniformly exponentially stable if there exists $\beta(\alpha)>0$ such that for $\alpha$ fixed all solutions satisfy an inequality of the form

$$
\left|x_{\alpha}(t, \phi)\right| \leq M \exp (-\beta(\alpha) t)|\phi|, \quad t \geq 0
$$

where $\phi:\left[-\alpha h_{m}, 0\right] \rightarrow R^{n}$ is continuous,

$$
|\phi|=\sup \left\{|\phi(t)|: t \in\left[-\alpha h_{m}, 0\right]\right\},
$$

and

$$
x_{\alpha}(t, \phi)=\phi(t) \quad \text { on }\left[-\alpha h_{m}, 0\right] .
$$

THEOREM 2.5. If $\sigma\left(G_{\alpha}\right)<0$ on $[a, b)$, then every member of the family (2.26) is uniformly exponentially stable.

Proof. The proof of this statement is due to D. Henry [7].

3. Some applications. In this section we shall apply the results of Sec. 2 to show how the exponential stability of autonomous retarded and neutral differential-difference equations can sometimes be inferred from a knowledge that related ordinary differential equations are exponentially stable. The first result of this section is a consequence of Theorem 2.1 and properties of linear retarded functional differential equations.

THEOREM 3.1. Let

$$
F_{\alpha}(s)=s I-\sum_{j=0}^{m} A_{j} \exp \left(-\alpha s h_{j}\right)
$$

and assume $\sigma\left(F_{0}\right)<0$. Then there exists a maximal interval $[0, q), q>0$, such that $\sigma\left(F_{\alpha}\right)<$ 0 on $[0, q)$ and hence for all $\alpha$ on this interval $(2.25)$ is exponentially stable. If $q<\infty, \sigma\left(F_{q}\right)$ $=0$ and system (2.25) has a periodic solution for $\alpha=q$.

Proof. Since, by Theorem 2.1, $\sigma\left(F_{\alpha}\right)$ is a continuous real-valued function on $R^{+}$and $\sigma\left(F_{0}\right)<0$ it follows that there exists a largest relatively open connected set in $R^{+},[0, q), q$ $>0$, such that $\sigma\left(F_{(0, q)}\right) \subset(-\infty, 0)$. By Theorem 2.4 this implies that every member of, (2.25) with $\alpha \in[0, q)$ is exponentially stable. If $q<\infty$, then by the maximal property of the interval $[0, q)$ it follows that $\sigma\left(F_{q}\right)=0$. But for linear autonomous retarded differential equations it is known that $\sigma\left(F_{\alpha}\right)$ is always the real part of a root of the $\operatorname{det} F_{\alpha}(s)=0$ (see e.g. [6]). Hence $\operatorname{det} F_{q}(s)=0$ has a root of the form $s=i \omega, \omega$ real. Again from the theory of functional differential equations [6], this implies that for $\alpha=q$ there exists a nontrivial solution of $(2.25)$ of period $\omega$.

Theorem 3.2. Assume det $\left[I-\sum_{j=1}^{m} B_{j} \exp \left(-s h_{j}\right)\right]=0$ has all roots in some closed half plane $\left(-\infty,-\beta_{0}\right], \beta_{0}>0$, i.e. $\sigma\left(B_{1}\right) \leq-\beta_{0}$. Let

$$
G_{\alpha}(s)=s\left(I-\sum_{j=1}^{m} B_{j} \exp \left(-\alpha s h_{j}\right)\right)-\sum_{j=0}^{m} A_{j} \exp \left(-\alpha s h_{j}\right)
$$

and assume $\sigma\left(G_{0}\right)<0$. Then there exists a maximal interval $[0, q), q>0$, such that $\sigma\left(G_{\alpha}\right)<$ 
0 on $[0, q)$ and hence for all $\alpha$ on this interval $(2.26)$ is uniformly exponentially stable. If $q$ $<\infty, \sigma\left(G_{q}\right)=0$ and the system (2.26) has a periodic solution for $\alpha=q$.

Proof. By Theorems 2.2 and $2.3 \sigma\left(G_{\sigma}\right)$ is continuous on $[0, \infty)$. Hence since $\sigma\left(G_{0}\right)<0$ there is a largest relatively open connected set in $R^{+},[0, q)$, such that $\sigma\left(G_{\mid 0, q)}\right) \subset(-\infty, 0)$. By Theorem 2.5 every member of (2.26) with $\alpha \in[0, q)$ is uniformly exponentially stable. If $q<\infty$, then $\sigma\left(G_{q}\right)=0$. This implies, since $\sigma\left(B_{q}\right) \leq-\beta_{0} / q<0$, that $\sigma\left(G_{q}\right)=\sigma\left(A_{q}\right)$. By Remark 2.1 this implies that there exists $i \omega, \omega$ real, such that $\operatorname{det} G_{q}(i \omega)=\operatorname{det} A_{q}(i \omega)=0$. From the general theory of neutral functional differential equations this implies the existence for $\alpha=q$ of a nontrivial periodic solution of (2.26) of period $\omega$.

The next two theorems give sufficient conditions which guarantee that (2.25) and (2.26) are uniformly exponentially stable for all values of $\alpha$.

THEOREM 3.3. If $\sum_{j=0}^{m} \boldsymbol{A}_{j}$ is Hurwitzian and

$$
\operatorname{det}\left[i \omega I-\sum_{j=0}^{m} A_{j} \exp \left(-i \omega \alpha h_{j}\right)\right]=0
$$

has no solution for $\omega$ real and $\alpha \geq 0$, then for all $\alpha \geq 0$ (2.25) is exponentially stable.

Proof. The proof is an immediate consequence of Theorem 3.1 since the nonexistence of real solutions of (3.3) guarantees $\sigma\left(F_{\alpha}\right) \neq 0$ for any $\alpha$. On the other hand, $\sum_{j=0}{ }^{m} A_{j}$ being Hurwitzian guarantees that $\sigma\left(F_{0}\right)<0$. Hence $\sigma\left(F_{(0, \infty)}\right) \subset(-\infty, 0)$, which proves the theorem.

THEOREM 3.4. If det $\left[I-\sum_{j=1}^{m} B_{j} \exp \left(-s h_{j}\right)\right]=0$ has all solutions in $\left(-\infty,-\beta_{0}\right], \beta_{0}>0,(I$ $\left.-\sum_{j=1}^{m} B_{j}\right)^{-1} \sum_{j=0}^{m} A_{j}$ is Hurwitzian and det $G_{\alpha}(i \omega) \neq 0$ for all $\alpha, \omega \geq 0$, then (2.26) is uniformly exponentially stable for all $\alpha \geq 0$.

Proof. The first condition of the theorem guarantees, because of Theorems 2.2 and 2.3, that $\sigma\left(G_{\alpha}\right)$ is continuous on $[0, \infty)$. The second condition states that $\sigma\left(G_{0}\right)<0$ and the third that $\sigma\left(G_{q}\right) \neq 0$ for any $q$ in $R^{+}$. Hence by Theorem 3.2 the system (2.26) is uniformly exponentially stable for all $\alpha \geq 0$.

Example 3.1. Let

$$
A_{0}=\left(\begin{array}{rr}
-1 & 0 \\
0 & -1
\end{array}\right)
$$

and

$$
A_{1}=\left(\begin{array}{rr}
0 & 1 \\
-1 & 0
\end{array}\right)
$$

Consider the family of delay differential equations of the form

$$
\dot{x}(t)=A_{0} x(t)+A_{1} x(t-h) .
$$

For $h=0(3.4)$ is exponentially stable. Let us compute the value of $q$, if it exists, for which $[0, q)$ is the maximal interval described in Theorem 3.3. Thus we seek $\omega$ real and $q>0$ such that

$$
\operatorname{det}\left[i \omega I-A_{0}-A_{1} \exp (-i \omega q)\right]=0=\left(i \omega^{2}+1\right)+\exp (-2 i \omega q) .
$$

Equating real and imaginary parts of (3.5), we arrive at the equations

$$
\begin{gathered}
-\omega^{2}+1=-\cos 2 \omega q, \\
2 \omega=\sin 2 \omega q .
\end{gathered}
$$


It is easily verified that (3.6) and (3.7) have no common solution for $\omega$ real. This implies, by Theorem 3.4, that (3.4) is exponentially stable for all nonnegative values of $h$.

Example 3.2. Let us alter (3.4) slightly in that we assume

$$
A_{1}=\left(\begin{array}{rr}
0 & \tau \\
-\tau & 0
\end{array}\right) \text { where } \tau>1 \text {. }
$$

Then (3.5) becomes

$$
(i \omega+1)^{2}+\tau^{2} \exp (-2 i \omega q)=0 .
$$

Separating real and imaginary parts of the above equation, we obtain

$$
\omega^{2}-1=\tau^{2} \cos ^{2} \omega q, \quad 2 \omega=\tau^{2} \sin \omega q .
$$

The positive real solution of the above equations for $\omega$ is

$$
\omega=\tau-1 \text {. }
$$

Then with a little manipulation we obtain

$$
q=\frac{1}{\tau-1} \tan ^{-1} \frac{2 \tau}{\tau^{2}-1}
$$

as our value for $q$ which satisfies Theorem 3.1 .

Example 3.3. Consider the neutral system which satisfies the hypotheses of Theorem 3.2:

$$
\frac{d}{d t}[x(t)-B x(t-h)]=A_{0} x(t)+A_{1} x(t-h)
$$

where

$$
B=\left(\begin{array}{rr}
0 & \frac{1}{2} \\
-\frac{1}{2} & 0
\end{array}\right), \quad A_{0}=\left(\begin{array}{rr}
-1 & 0 \\
0 & -1
\end{array}\right), \quad A_{1}=\left(\begin{array}{rr}
0 & 1 \\
-1 & 0
\end{array}\right)
$$

For $h=0$ it can be easily verified that the system is exponentially stable, since $(I-B)^{-1}$ $\left(A_{0}+A_{1}\right)$ is Hurwitzian. Furthermore, the eigenvalues of $B$ are $\pm i / 2$, and hence inside $|s|<1$. We could repeat the procedure used in Example 3.1 to seek $q$ such that det $G_{q}(i \omega)$ $=0$. However, this leads to an expression which required more analysis that this example warrants. Instead let

$$
y=\left(\begin{array}{rr}
1 & 1 \\
i & -i
\end{array}\right) x
$$

Then (3.8) transforms into

$$
\frac{d}{d t}[y(t)-\tilde{B} y(t-h)]=\tilde{A_{0}} y(t)+\tilde{A_{1}} y(t-h)
$$

where

$$
\tilde{B}=\left(\begin{array}{cc}
\frac{1}{2} i & 0 \\
0 & -\frac{1}{2} i
\end{array}\right), \quad \tilde{A_{0}}=A_{0}, \quad \tilde{A_{1}}=\left(\begin{array}{cc}
i & 0 \\
0 & -i
\end{array}\right) \text {. }
$$

Clearly the stability properties of (3.8) and (3.10) are the same. For (3.10) with $\omega$ real we 
obtain

$$
\begin{aligned}
\operatorname{det} G_{q}(i \omega)=0= & {\left[i \omega\left(1-\frac{1}{2} i \exp (-i \omega q)\right)+1-i \exp (-i \omega q)\right] } \\
& \times\left[i \omega\left(1+\frac{1}{2} i \exp (-i \omega q)\right)+1+i \exp (-i \omega q)\right] .
\end{aligned}
$$

Separating real and imaginary parts of the first factor in (3.11), we obtain

$$
\begin{gathered}
\frac{\omega}{2} \cos \omega q+1=\sin \omega q, \\
\omega\left[1-\frac{\sin \omega q}{2}\right]=\cos \omega q .
\end{gathered}
$$

From the second factor in (3.11) we obtain the equations

$$
\begin{gathered}
-\frac{\omega}{2} \cos \omega q+1=-\sin \omega q, \\
\omega\left[1+\frac{1}{2} \sin \omega q\right]=-\cos \omega q .
\end{gathered}
$$

After first verifying that $\cos q=0$ is not a solution of (3.12) and (3.13) we eliminate $\omega$ to obtain

$$
-(2-\sin \omega q)(1-\sin \omega q)=1-\sin ^{2} \omega q
$$

or $\sin \omega q=1$. But this is impossible; hence (3.12) and (3.13) have no solution for real $\omega$.

Examining (3.14) and (3.15) we see that $\cos \omega q=0$ is not a solution. Eliminating $\omega$ between these two equations, we obtain

$$
2 \sin ^{2} \omega q-\sin \omega q-3=0
$$

or $\sin \omega q=-1$, which is again impossible. Hence the system (3.10), or what is the same (3.8), is uniformly asymptotically stable for all $h \geq 0$.

Example 3.4. Let the system be given by Eq. (3.10) where $\tilde{A}_{0}$ and $\tilde{B}$ are as in Example 3.3 but $\tilde{A_{1}}$ is the matrix

$$
\tilde{A_{1}}=\left(\begin{array}{cc}
\tau i & 0 \\
0 & -\tau i
\end{array}\right), \quad \tau>1 .
$$

Again $\sigma\left(G_{0}\right)$ is Hurwitzian and $\sigma(B)=\frac{1}{2}$; hence $\sigma[I-B \exp (-s)]=-\ln 2<0$. Thus the hypotheses of Theorem 3.2 are satisfied and

$$
\begin{aligned}
\operatorname{det} G_{q}(i \omega)=0= & {\left[i \omega\left(1-\frac{i}{2} \exp (-i \omega q)\right)+1-\tau i \exp (-i \omega q)\right] } \\
& \times\left[i \omega\left(1+\frac{1}{2} \exp (-i \omega q)\right)+1+\tau i \exp (-i \omega q)\right]
\end{aligned}
$$

Equating real and imaginary parts of both factors leads to the two pairs of equations

$$
\begin{gathered}
\frac{\omega}{2} \cos \omega q+1=\tau \sin \omega q, \\
\omega\left[1-\frac{1}{2} \sin \omega q\right]=\tau \cos \omega q,
\end{gathered}
$$

and

$$
-\frac{\omega}{2} \cos \omega q+1=-\tau \sin \omega q,
$$




$$
\omega\left[1+\frac{1}{2} \sin \omega q\right]=-\tau \cos \omega q .
$$

Elementary calculations on (3.16)-(3.17) lead to the solutions

$$
\sin \omega q=\frac{2 \tau+1}{4 \tau+2}, \quad \cos \omega q=\frac{\left(3\left(\tau^{2}-1\right)\right)^{1 / 2}}{2 \tau+1}
$$

and

$$
\omega=\frac{2(3)^{1 / 2}}{3}\left(\tau^{2}-1\right)^{1 / 2} .
$$

Thus $G_{q}(i \omega)=0$ has a solution for some $q<\infty$ and $\omega$ real if $\tau>1$. Similarly calculations on (3.18)-(3.19) yield the negative values of $\omega$ and $\sin \omega q$ expressed by (3.20)-(3.21).

Example 3.5. As a final example we shall show that a system of the form

$$
\frac{d}{d t}[x(t)-B x(t-h)]=A x(t)
$$

where $A$ is Hurwitzian and $\sigma(B)<1$, i.e. $\sigma(I-B \exp (-s))<0$, can satisfy the hypotheses of Theorem 3.2 and have a maximal interval of uniform exponential stability $[0, q)$, with $q$ $<\infty$.

Consider (3.22) where

$$
A=\left(\begin{array}{rr}
0 & 1 \\
-\frac{5}{4} & -1
\end{array}\right), \quad B=\left(\begin{array}{ll}
\frac{1}{2} & 0 \\
0 & \frac{1}{2}
\end{array}\right) .
$$

It is trivial to verify that the system (3.22) with $A$ and $B$ given above satisfy the hypotheses of Theorem 3.2. Furthermore,

$$
\begin{aligned}
\operatorname{det}[i \omega(I-B & \exp (-i \omega q))-A]=0 \\
& =\left[i \omega\left(1-\frac{1}{2} \exp (-i \omega q)\right)+\frac{1}{2}-i\right]\left[i \omega\left(1-\frac{1}{2} \exp (-i \omega q)+\frac{1}{2}+i\right] .\right.
\end{aligned}
$$

The above equation leads to two systems of equations

$$
\begin{aligned}
& -\frac{\omega}{2} \sin \omega q+\frac{1}{2}=0, \\
& \omega\left(1-\frac{1}{2} \cos \omega q\right)=1,
\end{aligned}
$$

and

$$
\begin{aligned}
& -\frac{\omega}{2} \sin \omega q+\frac{1}{2}=0, \\
& \omega\left(1-\frac{1}{2} \cos \omega q\right)=-1 .
\end{aligned}
$$

The solution of both sets of equations is $\omega=1, \sin \omega q=1$. Thus the smallest value of $q$ which satisfies Theorem 3.2 is $q=\pi / 2$.

\section{REFERENCES}

[1] A. S. Besicovitch, Almost periodic functions, Dover Publications, Inc., 1954

[2] H. Bohr, Collected Mathematical Works, Vol. 1, Dansk Matemetisk Forening, København, 1952. See also H. 
Bohr, Über die gleich Mässige Konvergenz Dirichletsher Reihen, J. Reine Angew. Math. 143, 203-211(1913)

[3] R. Datko, Representation and stability of linear differential-difference equations in a Banach space, to appear.

[4] R. Datko, The stabilization of linear functional differential equations, in Calculus of variations and control theory, ed. D. L. Russell, Academic Press, Inc., New York, 1975, pp. 353-369

[5] L. E. El'sgol'ts and S. B. Norkin, Introduction to the theory and application of differential equations with deviating arguments, Academic Press, New York, 1973

[6] J. Hale, Theory of functional differential equations, Springer-Verlag, New York, 1977

[7] D. Henry, Linear autonomous neutral functional differential equations, J. Diff. Eqs. 15, 106-128 (1974)

[8] S. Saks and A. Zygmund, Analytic functions, Elsevier Publishing Co., New York, 1971 